\title{
The Correlation of Calculated Testosterone Indices with Metabolic Markers in Polycystic Ovarian Syndrome
}

\author{
(1) Nil Atakul, (1) Berna Șermin Kılıç \\ Department of Gynecology and Obstetrics, İstanbul Training and Research Hospital, İstanbul, Turkey
}

\section{Abstract}

BACKGROUND/AIMS: Polycystic ovarian syndrome (PCOS) is mainly considered a reproductive disease with chronic anovulation and infertility. Metabolic syndrome risk in patients with PCOS is shown in many studies, and mainly hyperandrogenemia component has a strong predictor of metabolic disorders. This study aimed to compare the value of bioavailable testosterone (BioT) and free androgen index (FAI) levels to determine the correlation with metabolic parameters in patients with PCOS.

MATERIALS and METHODS: This study included 83 females with PCOS of reproductive age, according to Rotterdam criteria from outpatient clinics of the Department of İstanbul Training and Research Hospital from January 2020 to October 2020 for our retrospective cohort analysis. FAI was calculated as FAI $=($ testosterone/SHBG $) \times 100$. Calculations of BioT were conducted using the formula from total testosterone $(\mathrm{TT}), \mathrm{SHBG}$, and albumin values.

RESULTS: FAI and BioT levels were $5.60 \pm 5.73 \mathrm{ng} / \mathrm{dL}$ and $21.11 \pm 15.44 \mathrm{ng} / \mathrm{dL}$ respectively. A positive, moderate, and statistically significant correlation was found between body mass index (BMI) and FAI ( $r=0.402, p=0.009)$, whereas a positive, moderate, and statistically significant correlation between BMI and BioT $(r=0.491, p=0.002)$. A positive, moderate, and statistically significant correlation was found between lowdensity lipoprotein and $\mathrm{FAI}(r=0.377, p=0.040)$.

CONCLUSION: Our study is one of the few studies that investigated and compared the concentrations of BioT and FAI with the metabolic markers in PCOS and indicated the corresponding suitability of both androgen indices in detecting metabolic syndrome risk in PCOS. These indices give more valuable insight into metabolic disturbances than commonly analyzed TT levels.

Keywords: Polycystic ovary syndrome, metabolic syndrome, hyperandrogenism

\section{INTRODUCTION}

Polycystic ovarian syndrome (PCOS) is a common disease of females, associated with chronic anovulation. Hyperandrogenism is the key feature of PCOS as defined by all criteria. ${ }^{1-3}$

Patients with PCOS have a most commonly determined hyperandrogenism by calculating the total testosterone (TT) levels, but low concentrations in women typically lead to poor diagnostic performance; however, its measurement is questionable. ${ }^{4}$ PCOS has mainly been considered a reproductive disorder; however, previous study results have shown that PCOS also has an association with metabolic and cardiovascular risk. ${ }^{5,6}$

Albumin and sex hormone-binding globulin (SHBG) are two of the four proteins that bind to circulated plasma testosterone

To cite this article: Atakul N, Kılıç BŞ. The Correlation of Calculated Testosterone Indices with Metabolic Markers in Polycystic Ovarian Syndrome. Cyprus J Med Sci 2021;6(Suppl 1):63-68

ORCID iDs of the authors: N.A. 0000-0003-3874-8797; B.S.K. 0000-0002-0103-0709. 
in the bloodstream. Only $0.5 \%-3 \%$ of TT is not protein-bound and free, referred to as free testosterone (FT). The testosterone bound by albumin, also known as "weakly bound" testosterone, immediately detaches, and becomes bioavailable when the FT levels are decreased. Thus, the sum of FT and albumin-bound are collectively referred to as the "bioavailable" testosterone (BioT), this is the percentage of testosterone that readily enters the cells and can be used for signaling. ${ }^{7,8}$

Moreover, gene expression and cell function regulation, and intracellular androgen receptor interaction are mainly coordinated by BioT. ${ }^{9}$ Consequently, testosterone that is not bound to SHBG is commonly treated as biologically accessible to tissues; therefore, BioT may be a more accurate predictor of androgen bioactivity than TT. ${ }^{10,11}$

The sex hormone-bound fraction of testosterone is defined as the free androgen index (FAl) and is presumed to be intrinsically superior in hyperandrogenemia estimation, compared with TT measurement alone.

However, the definite contribution and aspect of BioT and FAI compared to TT or dehydroepiandrosterone sulfate (DHEA-SO4) in females with PCOS was not yet studied. Most current scientific reports on the clinical consequences of hyperandrogenism in patients with PCOS have focused on TT, whereas knowledge about the relationship of BioT and FAI levels with associated adverse outcomes is limited.

This study aimed to compare the values of different androgen markers with metabolic parameters in patients with PCOS.

\section{MATERIALS and METHODS}

This study included 83 females of reproductive age with PCOS for the retrospective cohort analysis from the outpatient clinic of the Department of Istanbul Training and Research Hospital from January to October 2020. The Revised Rotterdam criteria were used in the diagnosis of patients with PCOS, requiring at least two of the three clinical and/or biochemical manifestations: (1) oligo- or anovulation; (2) hyperandrogenism (clinical and/or biochemical); and (3) polycystic ovaries. ${ }^{3}$

Patients who took medicines that affect the metabolic and hormonal parameters (androgen, anti-androgen, lipid, and glucose) six months before the study were excluded. The exclusion criteria included hypothyroidism, hyperprolactinemia, nonclassical 21-hydroxylase deficiency, Cushing's syndrome, and androgen-secreting tumors, as well as individuals with chronic diseases (including cardiovascular-renal diseases), malignancies, active infection, and regular drug/alcohol/cigarette usage.

\section{Laboratory Analysis}

Hormone levels and lipid markers were analyzed in a biochemical laboratory. The serum levels of follicle-stimulating hormone (FSH), luteinizing hormone (LH), TT, prolactin (PRL), DHEA-SO4, and thyroid-stimulating hormone (TSH) serum levels were assessed using a UniCel Dxl800 analyzer (Beckman Coulter, Brea, $\mathrm{CA}$ ). Anti-Mullerian hormone (AMH) levels were measured using the electrochemiluminescence immunoassay method (Roche Cobas E411, Roche Diagnostics, Mannheim, Germany). Metabolic and lipid profiles, fasting glucose, high-density lipoprotein (HDL), low-density lipoprotein (LDL), total cholesterol, and triglyceride were determined using a spectrophotometer (Beckman Coulter AU 5800 analyzer Beckman Coulter, Brea, CA, USA). The homeostasis model assessment for insulin resistance $($ HOMA-IR) was HOMA-IR $=$ fasting blood glucose $(\mathrm{mmol} / \mathrm{L}) \times$ fasting blood plasma insulin $(\mathrm{mU} / \mathrm{mL}) / 22.5$. SHBG concentrations were measured using an immunoassay (ARCHITECT ${ }^{\circ}$, Abbott Diagnostics).

The FAI was calculated as $\mathrm{FAI}=($ testosterone $/ \mathrm{SHBG}) \times 100$. Formula from the International Society for the Study of the Aging Male website was used for the BioT measurement (http:/www. issam.ch/freetesto.htm) from TT, albumin, and SHBG values that are calculated in the same manner described by Vermeulen et al. ${ }^{12}$

The study protocol was approved by the Ethics Committee of Istanbul Training and Research Hospital (date: 24/09/2020, issue number: 2054) and was conducted following the Declaration of Helsinki.

\section{Statistical Analysis}

Mean \pm standard deviation was used in the expression of continuous variables, whereas categorical data was given as numbers and percentages. In the intergroup analysis for continuous variables, the Kolmogorov-Smirnov test for univariate data was used to assess the normal distribution data. The oneway analysis of variance test was used for three groups when the data were suitable for normal distribution; a t test was used for two groups, whereas the Mann-Whitney $U$ test was used for two groups when the former was not suitable. The linear relationship between the variables was evaluated using Pearson's correlation test. Analyses were performed with International Business Machines Statistical Package for the Social Sciences Package Program (version 22.0; IBM Corporation, Armonk, NY, USA). Cases in which a type 1 error level of $<5 \%$ was considered statistically significant.

\section{RESULTS}

The mean age of patients with PCOS was $23.19 \pm 5.41$ years, and their mean body mass index (BMI) value was 25.91 6 6.23. AMH, LDL, HDL, total cholesterol, LH, FSH, PRL, TSH, fasting glucose, triglyceride, FT, hemoglobin A1c (HbA1c), HOMA-IR, estradiol (E2), SHBG, and insulin levels, and their mean and median values are presented in Table 1. TT, DHEA-SO4, FAI, and BioT levels were 
$65.26 \pm 25.41 \mathrm{ng} / \mathrm{dL}, 268.50 \pm 108.43 \mu \mathrm{g} / \mathrm{dL}, 5.60 \pm 5.73 \mathrm{ng} / \mathrm{dL}$, and $21.11 \pm 15.44 \mathrm{ng} / \mathrm{dL}$, respectively.

A negative, moderate, and statistically significant correlation was found between SHGB and FAI ( $r=-0.522, p<0.001)$, whereas a positive, moderate, and statistically significant correlation between TT and FAI ( $r=0.443, p=0.005)$.

A strong, negative, and statistically significant correlation was determined between SHGB and BioT $(r=-0.625, p<0.001)$. A positive, moderate, and statistically significant correlation was determined between TT and BioT ( $r=-0.625, p<0.001)$. 0.434, $p=0.006)$. A positive and statistically significant correlation $(r=0.877, p<0.001)$ was also found between FAI and BioT.

A negative, moderate, and statistically significant correlation was found between SHGB and DHEA SO4 ( $r=-0.401, p=0.015)$, whereas a positive, moderate, and statistically significant correlation between TT and DHEA SO4 ( $r=-0.401, p=0.015)$. $0.461, p=0.001)$. Furthermore, a positive, moderate, and statistically significant correlation was found between $\mathrm{FAI}$ and DHEA-SO4 ( $r=0.531, p=0.001$ ), whereas a positive and moderate correlation was found between BioT and DHEA SO4 ( $r=0.529$, $p=0.002$ ). A positive, moderate, and statistically significant correlation was found between BMI and FAI $(r=0.402, p=0.009)$, whereas a positive, moderate, and statistically significant correlation between BMI and BioT ( $r=0.491, p=0.002)$ (Table 2). A positive, moderate, and statistically significant correlation was found between LDL and FAI ( $r=0.377, p=0.040)$.

The total cholesterol and HOMA-IR values for FAI was $\geq 8.4 \mathrm{ng} / \mathrm{dL}$ in patients with PCOS, which was higher than the FAI of $<8.4 \mathrm{ng} /$ $\mathrm{dL}$ cut-off value; differences were not significant $(p=0.305$ and $p=0.088$ ). The total cholesterol and HOMA-IR values for TT was $\geq 75 \mathrm{ng} / \mathrm{dL}$, which was higher than the TT of $<75 \mathrm{ng} / \mathrm{dL}$ cut-off value; differences were significant only for HOMA-IR ( $p=0.326$ and $p=0.040$, respectively). The total cholesterol and HOMA-IR values for DHEA-SO4 was $\geq 391 \mu \mathrm{g} / \mathrm{dL}$, which was higher than the cut-off value for DHEA SO4 of $<391 \mu \mathrm{g} / \mathrm{dL}$; differences were not significant ( $p=0.051$ and $p=0.260$, respectively). The total cholesterol and HOMA-IR values for BioT was $\geq 10 \mathrm{ng} / \mathrm{dL}$, which was higher than the BioT of $<10 \mathrm{ng} / \mathrm{dL}$ cut-off value; differences were not significant $(p=0.638$ and $p=0.267$, respectively) (Table 3).

\section{DISCUSSION}

A correlation was found between the LDL and BioT, BMI with BioT and FAI, and HOMA-IR with TT levels of patients with PCOS. Our study revealed the relationship between FAI and BioT values and TT, DHEA-SO4, and SHBG levels. Our results indicate the measurements of FT indices that provide new information on metabolic disturbances in PCOS.
The studies that investigate the relationship of metabolic syndrome (MetS) with TT and SHBG levels have been inconsistent. The clinical findings associated with MetS include abdominal obesity, dyslipoproteinemia, impaired glucose tolerance, and hypertension. A recent cross-sectional study in men reported that a lower TT level is correlated with low HDL, abdominal obesity, and hypertension. FT levels are found to be correlated with hypertension, whereas SHBG shows no correlation. ${ }^{13}$ Another cross-sectional study also found a correlation between abdominal obesity and low TT levels. ${ }^{14}$

A limited number of reports are available on FAl levels in patients with PCOS and their associated consequences. Li et al..$^{15}$ found that high serum FAl levels were found in patients with a higher BMI and total cholesterol, HOMA-IR, and DHEA-SO4 levels. The 2005 SWAN study included 3,302 females and revealed low SHBG and high FAl levels associated with a higher BMI, triglycerides,

Table 1. Metabolic and Hormonal Parameters in Patients with PCOS

\begin{tabular}{|c|c|}
\hline & $P \operatorname{Cos}(n=83)$ \\
\hline Age (year) (Mean \pm SD) & $23.19 \pm 5.41$ \\
\hline BMI $\left(\mathrm{kg} / \mathrm{m}^{2}\right)($ Mean $\pm \mathrm{SD})$ & $25.91 \pm 6.23$ \\
\hline $\mathrm{AMH}(\mathrm{ng} / \mathrm{ml})($ Mean $\pm \mathrm{SD})$ & $7.82 \pm 5.17$ \\
\hline FSH (U/L) (Mean \pm SD) & $6.18 \pm 2.07$ \\
\hline LH (U/L) [Medyan (min-max)] & $6.7(0.4-27.8)$ \\
\hline Total testosterone $(\mathrm{ng} / \mathrm{dL})($ Mean $\pm \mathrm{SD})$ & $65.26 \pm 25.41$ \\
\hline DHEA-SO4 $(\mu \mathrm{g} / \mathrm{dL})($ Mean $\pm \mathrm{SD})$ & $268.50 \pm 108.43$ \\
\hline PRL $(\mu g / L)($ Mean $\pm S D)$ & $15.25 \pm 10.53$ \\
\hline TSH (ng/ml) (Mean \pm SD) & $1.82 \pm 0.81$ \\
\hline Fasting glucose $(\mathrm{mg} / \mathrm{dL})($ Mean $\pm \mathrm{SD})$ & $89.99 \pm 14.63$ \\
\hline Trigliceride $(\mathrm{mg} / \mathrm{dL})$ (Mean \pm SD) & $88.16 \pm 45.19$ \\
\hline LDL (mg/dL) (Mean \pm SD) & $112.20 \pm 32.59$ \\
\hline $\mathrm{HDL}(\mathrm{mIU} / \mathrm{mL})($ Mean $\pm \mathrm{SD})$ & $54.47 \pm 17.12$ \\
\hline Total cholesterol (mIU/mL) (Mean \pm SD) & $177.02 \pm 36.44$ \\
\hline Free Androgen Index (FAI) (ng/dL) (Mean \pm SD) & $5.60 \pm 5.73$ \\
\hline Bioavailable Testosterone (ng/dL) (Mean \pm SD) & $21.11 \pm 15.44$ \\
\hline Free Testosterone $(\mathrm{ng} / \mathrm{dL})($ Mean $\pm \mathrm{SD})$ & $0.91 \pm 0.71$ \\
\hline Hba1c (Mean \pm SD) & $5.48 \pm 0.44$ \\
\hline HOMA-IR Median (min-max) & $1.8(0.7-9.90)$ \\
\hline E2 (ng/L) Median (min-max) & $34(14-445)$ \\
\hline SHGB (nmol/L) Median (min-max) & $48.3(9.6-418)$ \\
\hline Fasting insulin (mU/L) Median (min-max) & $8.75(4-105)$ \\
\hline \multicolumn{2}{|c|}{$\begin{array}{l}\text { Values are described as mean } \pm \text { standard deviation, median (minimum- } \\
\text { maximum). }\end{array}$} \\
\hline \multicolumn{2}{|c|}{$\begin{array}{l}\text { BMI, Body mass index; PCOS, Polycystic ovarian syndrome; AMH, Anti-mullerian } \\
\text { hormone; LH, Luteinizing hormone; FSH, Follicle-stimulating hormone; PRL, } \\
\text { Prolactin; DHEA-SO4, Dehydroepiandrosterone sulfate; TSH, Thyroid-stimulating } \\
\text { hormone; HDL, High-density lipoprotein; LDL, Low-density lipoprotein; E2, } \\
\text { Estradiol; SHBG, Sex hormone-binding globulin; n, Number. }\end{array}$} \\
\hline
\end{tabular}




\begin{tabular}{|c|c|c|c|c|c|c|}
\hline & & SHGB (nmol/L) & $\begin{array}{l}\text { Total testosterone } \\
\text { (ng/dL) }\end{array}$ & $\begin{array}{l}\text { Free Androgen } \\
\text { Index (FAI) (ng/ } \\
\text { dL) }\end{array}$ & $\begin{array}{l}\text { Bioavailable } \\
\text { testosterone } \\
(\mathrm{ng} / \mathrm{dL})\end{array}$ & $\begin{array}{l}\text { DHEA S04 } \\
(\mu \mathrm{g} / \mathrm{dL})\end{array}$ \\
\hline \multirow{3}{*}{ Total Testosterone (ng/dL) } & $r$ & 0.108 & & & & \\
\hline & $p$ & 0.507 & & & & \\
\hline & $\mathrm{N}$ & 40 & & & & \\
\hline \multirow{3}{*}{$\begin{array}{l}\text { Free Androgen Index (FAI) (ng/ } \\
d L)\end{array}$} & $r$ & $-0.522(* *)$ & $0.443(* *)$ & & & \\
\hline & $p$ & $<0.001$ & 0.005 & & & \\
\hline & $\mathrm{N}$ & 41 & 39 & & & \\
\hline \multirow{3}{*}{$\begin{array}{l}\text { Bioavailable Testosterone } \\
\text { (ng/dL) }\end{array}$} & $r$ & $-0.625(* *)$ & $0.434(* *)$ & $0.877(* *)$ & & \\
\hline & $p$ & $<0.001$ & 0.006 & $<0.001$ & & \\
\hline & $\mathrm{N}$ & 39 & 38 & 39 & & \\
\hline \multirow{3}{*}{$\begin{array}{l}\text { DHEA-SO4 } \\
(\mu \mathrm{g} / \mathrm{dL})\end{array}$} & $r$ & $-0.401\left(^{*}\right)$ & $0.461(* *)$ & $0.531(* *)$ & $0.529(* *)$ & \\
\hline & $p$ & 0.015 & 0.001 & 0.001 & 0.002 & \\
\hline & $\mathrm{N}$ & 36 & 53 & 35 & 33 & \\
\hline \multirow{3}{*}{ LDL (mg/dL) } & $r$ & -0.134 & 0.138 & $0.377(*)$ & 0.340 & 0.220 \\
\hline & $p$ & 0.450 & 0.346 & 0.040 & 0.076 & 0.132 \\
\hline & $\mathrm{N}$ & 34 & 49 & 30 & 28 & 48 \\
\hline \multirow{3}{*}{$\begin{array}{l}\mathrm{HDL} \\
(\mathrm{mIU} / \mathrm{mL})\end{array}$} & $r$ & 0.161 & 0.023 & -0.291 & -0.338 & -0.031 \\
\hline & $p$ & 0.320 & 0.870 & 0.090 & 0.059 & 0.823 \\
\hline & $\mathrm{N}$ & 40 & 55 & 35 & 32 & 53 \\
\hline \multirow{3}{*}{$\begin{array}{l}\text { Triglyceride } \\
\text { (mg/dL) }\end{array}$} & $r$ & -0.020 & 0.095 & 0.178 & 0.147 & 0.008 \\
\hline & $p$ & 0.900 & 0.489 & 0.299 & 0.415 & 0.955 \\
\hline & $\mathrm{N}$ & 41 & 55 & 36 & 33 & 53 \\
\hline \multirow{3}{*}{$\begin{array}{l}\text { Fasting glucose } \\
(\mathrm{mg} / \mathrm{dL})\end{array}$} & $r$ & -0.037 & 0.014 & 0.035 & 0.050 & 0.156 \\
\hline & $p$ & 0.811 & 0.921 & 0.834 & 0.768 & 0.264 \\
\hline & $\mathrm{N}$ & 45 & 56 & 39 & 37 & 53 \\
\hline \multirow{3}{*}{$\begin{array}{l}\text { Fasting insulin } \\
(\mathrm{mU} / \mathrm{L})\end{array}$} & $r$ & 0.005 & 0.032 & 0.014 & -0.033 & -0.162 \\
\hline & $p$ & 0.973 & 0.814 & 0.935 & 0.849 & 0.242 \\
\hline & $\mathrm{N}$ & 43 & 56 & 38 & 36 & 54 \\
\hline \multirow{3}{*}{ HOMA-IR } & $r$ & -0.086 & 0.165 & 0.232 & 0.196 & 0.039 \\
\hline & $p$ & 0.576 & 0.211 & 0.145 & 0.233 & 0.777 \\
\hline & $\mathrm{N}$ & 45 & 59 & 41 & 39 & 56 \\
\hline \multirow{3}{*}{ BMI $\left(\mathrm{kg} / \mathrm{m}^{2}\right)$} & $r$ & -0.199 & 0.150 & $\left.0.4022^{* *}\right)$ & $\left.0.4911^{* *}\right)$ & -0.029 \\
\hline & $p$ & 0.200 & 0.253 & 0.009 & 0.002 & 0.829 \\
\hline & $\mathrm{N}$ & 43 & 60 & 41 & 38 & 57 \\
\hline
\end{tabular}

fasting plasma glucose, and low HDL levels in premenopausal females. ${ }^{16}$ Similarly, our study revealed a positive correlation between BMI and LDL levels. These positive associations between FAI and LDL were also demonstrated in another report by Cai et al. $^{17}$, which found a positive association between FAl levels and LDL, BMI, waist circumference, and a negative association with HDL levels in females with PCOS. In line with our findings, non-SHBG-binding testosterone (BioT, FAI) levels were found to be correlated with obesity in patients with PCOS and healthy subjects. 


\begin{tabular}{|c|c|c|c|c|}
\hline $\begin{array}{l}\text { Free Androgen Index (FAI) } \\
<8.4 \mathrm{ng} / \mathrm{dL}(\mathrm{n}=53) \\
\geq 8.4 \mathrm{ng} / \mathrm{dL}(\mathrm{n}=30)\end{array}$ & $\begin{array}{l}174.20 \pm 32.80 \\
187.71 \pm 18.73\end{array}$ & $0.305^{*}$ & $\begin{array}{l}1.70(0.7-9.90) \\
2.25(1.50-5.80)\end{array}$ & $0.088^{* *}$ \\
\hline $\begin{array}{l}\text { DHEA-SO4 } \\
<391 \mu \mathrm{g} / \mathrm{dL}(\mathrm{n}=65) \\
\geq 391 \mu \mathrm{g} / \mathrm{dL}(\mathrm{n}=18)\end{array}$ & $\begin{array}{l}172.22 \pm 30.01 \\
191.00 \pm 17.50\end{array}$ & $0.051^{*}$ & $\begin{array}{l}1.70(0.7-9.90) \\
1.85(1.50-5.90)\end{array}$ & $0.260^{* * *}$ \\
\hline
\end{tabular}

The positive correlation between LDL and FAI is assumed as an indicator of high levels of FAl and is a good prognostic marker for patients with PCOS since high LDL levels are strongly associated with coronary artery diseases and cardiovascular risk.

Hyperinsulinemia stimulates the production of adrenal androgens, ${ }^{18}$ whereas SHBG levels decrease due to increased insulin levels through the inhibition of liver synthesis, which in turn, increases testosterone concentrations. ${ }^{19} \mathrm{~A}$ recent study used the HOMA-IR cut-off value of two to separate patients with PCOS into two groups and two-fold higher FAl values were found in the $>2$ HOMA-IR group than in the $<2$ HOMA-IR group. ${ }^{20}$ Insufficient correlation between fasting insulin, fasting glucose, and HOMA-IR levels with FAI and BioT in our study may be due to the relatively young age and low BMI of our study population. We assume that the FAI value in PCOS should be evaluated based on the different disease phenotypes.

Our study revealed a 5.6 mean FAI value in patients with PCOS. These results differed from those obtained in studies regarding PCOS in people of different ethnicities. In Malaysian females with PCOS, the mean FAl value was 9.2:21 6.1 among Chinese females, ${ }^{22}$ and 4.97 in a study on the European population. ${ }^{23}$ Standardized measurement methods and ethnicity-specific FAI cut-off levels should be investigated for the homogeneity of scientific findings through the establishment of an individual reference range and a validated assay.

This study had several limitations. First, this study was conducted using a relatively young and low BMI PCOS population; studies with wide phenotypic variation may provide more accurate and definitive results regarding the value of free androgen levels in these patients; however, measuring free androgen levels is still more definitive in determining the risk of MetS than using
TT and DHEA-SO4 levels for them. A relatively small number of studies in females with PCOS were recognized that investigate high androgen parameters and long-term health risks.

Therefore, more reports are needed with large and more diverse subjects with different populations that are observed for a longer period are mandatory to validate and confirm our study results and interpret the effects of different testosterone measurements in the risk for MetS and MetS associated increased risk of type 2 diabetes mellitus and cardiovascular disease in patients with PCOS.

\section{Main points}

- High FAI and BioT is a stronger predictor of MetS risk than other common androgenic parameters in females with PCOS.

- Patients with PCOS with high FAI and BioT levels should be carefully monitored by physicians for future metabolic consequences even if they had normal androgen parameter levels.

- FAl and BioT could be used to monitor the responsiveness to therapy of patients with PCOS instead of measuring the declined TT levels.

\section{ETHICS}

Ethics Committee Approval: The research was approved by the clinical research Ethics Committee of İstanbul Training and Research Hospital (decision no: 2054, date: 24.09.2020).

Informed Consent: Retrospective study.

Peer-review: Externally peer-reviewed. 


\section{Authorship Contributions}

Conception: N.A.; Design: N.A.; Materials: B.S..K.; Data Collection and/or Processing: N.A.; Analysis and/or Interpretation: N.A.; Statistical Analysis: B.S..K.; Writing: B.Ş.K.

\section{DISCLOSURES}

Financial Disclosure: The author declared that this study had received no financial support.

Conflict of Interest: The authors declare no conflict of interest.

\section{REFERENCES}

1. Bani MM, Majdi SA. Polycystic ovary syndrome (PCOS), diagnostic criteria, and AMH. Asian Pac J Cancer Prev. 2017;18:17-21.

2. Azziz R, Carmina E, Dewailly D, et al. Positions statement: criteria for defining polycystic ovary syndrome as a predominantly hyperandrogenic syndrome: an Androgen Excess Society guideline. J Clin Endocrinol Metab. 2006;91:4237-4245.

3. Rotterdam ESHRE/ASRM-Sponsored PCOS consensus workshop group. Revised 2003 consensus on diagnostic criteria and long-term health risks related to polycystic ovary syndrome (PCOS). Hum Reprod. 2004;19:41-47.

4. Luque-Ramírez M, Jiménez-Mendiguchia L, García-Cano A, et al. Certified testosterone immunoassays for hyperandrogenaemia. Eur J Clin Invest. 2018;48:e13029.

5. Wekker V, van Dammen L, Koning A, et al. Long-term cardiometabolic disease risk in women with PCOS: a systematic review and meta-analysis. Hum Reprod Update. 2020;26:942-960.

6. Oliver-Williams C, Vassard D, Pinborg A, Schmidt L. Risk of cardiovascular disease for women with polycystic ovary syndrome: results from a national Danish registry cohort study. Eur J Prev Cardiol. 2020;28:e39-41.

7. Philis $\mathrm{Y}$, Soyez M. Comparison between calculated bioavailable testosterone level with radio-immunoassay result. J Biol Today's World. 2021;10:1-5.

8. Stanczyk FZ, Matharu H, Winer SA. Bioidentical hormones. Climacteric. 2021;24:38-45.

9. Asthana S, Bhasin S, Butler RN, et al. Masculine vitality: pros and cons of testosterone in treating the andropause. I Gerontol A Biol Sci Med Sci. 2004;59:461-465.

10. Ramachandran S, Hackett GI, Strange RC. Sex hormone binding globulin: a review of its interactions with testosterone and age, and its impact on mortality in men with type 2 diabetes. Sex Med Rev. 2019;7:669-668.
11. Giton F, Guéchot J, Fiet J. Comparative determinations of non SHBG-bound serum testosterone, using ammonium sulfate precipitation, concanavalin A binding or calculation in men. Steroids. 2012;77:1306-1311.

12. Vermeulen A, Verdonck L, Kaufman JM. A critical evaluation of simple methods for the estimation of free testosterone in serum. J Clin Endocrinol Metab. 1999;84:3666-3672.

13. Moon H, Choi I, Kim S, et al. Cross-sectional association between testosterone, sex hormone-binding globulin and metabolic syndrome: the healthy twin study. Clin Endocrinol. 2017;87:523-531.

14. Blaya R, Thomaz LD, Guilhermano F, et al. Total testosterone levels are correlated to metabolic syndrome components. Aging Male. 2016;19:85-89.

15. Li H, Xu X, Wang X, et al. Free androgen index and Irisin in polycystic ovary syndrome. J Endocrinol Invest. 2016;39:549-556.

16. Sutton-Tyrrell K, Wildman RP, Matthews KA, et al. SWAN Investigators. Sex-hormone-binding globulin and the free androgen index are related to cardiovascular risk factors in multiethnic premenopausal and perimenopausal women enrolled in the Study of Women Across the Nation (SWAN). Circulation. 2005;111:1242-1249.

17. Cai J, Wu CH, Zhang Y, et al. High-free androgen index is associated with increased risk of non-alcoholic fatty liver disease in women with polycystic ovary syndrome, independent of obesity and insulin resistance. Int J Obes (Lond). 2017;41:1341-1347.

18. Unluhizarci K, Karaca Z, Kelestimur F. Role of insulin and insulin resistance in androgen excess disorders. World J Diabetes. 2021;12:616-629.

19. Qu X, Donnelly R. Sex hormone-binding globulin (SHBG) as an early biomarker and therapeutic target in polycystic ovary syndrome. Int J Mol Sci. 2020;21:8191.

20. Ożga K, Krzyczkowska-Sendrakowska M, Hubalewska-Dydejczyk A, et al. The value of the free androgen index depends on the phenotype of polycystic ovary syndrome-a single-centre experience. Endokrynol Pol. 2019;70:330335.

21. Nadaraja RND, Sthaneshwar P, Razali N. Establishing the cut off values of androgen markers in the assessment of polycystic ovarian syndrome. Malays J Pathol. 2018;40:33-9.

22. Zhou Z, Ni R, Hong Y, et al. Defining hyperandrogenaemia according to the free androgen index in Chinese women: a cross-sectional study. Clin Endocrinol (Oxf). 2012; 77:446-452.

23. Hahn S, Kuehnel W, Tan S, et al. Diagnostic value of calculated testosterone indices in the assessment of polycystic ovary syndrome. Clin Chem Lab Med. 2007; 45:202-207. 